\title{
Correction to: Clinicians' use of breast cancer risk assessment tools according to their perceived importance of breast cancer risk factors: an international survey
}

\author{
Anne Brédart ${ }^{1,2}$ - Jean-Luc Kop ${ }^{3}$ - Antonis C. Antoniou ${ }^{4}$ - Alex P. Cunningham ${ }^{4}$ - Antoine De Pauw ${ }^{5}$. \\ Marc Tischkowitz ${ }^{6} \cdot$ Hans Ehrencrona $^{7} \cdot$ Marjanka K. Schmidt ${ }^{8} \cdot$ Sylvie Dolbeault ${ }^{1,9} \cdot$ Kerstin Rhiem $^{10}$. \\ Douglas F. Easton ${ }^{4} \cdot$ Peter Devilee $^{11} \cdot$ Dominique Stoppa-Lyonnet $^{5} \cdot$ Rita Schmutzler $^{10}$
}

Published online: 22 July 2019

(C) Springer-Verlag GmbH Germany, part of Springer Nature 2019

Correction to: Journal of Community Genetics (2019)

10:61-71

https://doi.org/10.1007/s12687-018-0362-8

The published online version contains mistake in author list. The correct presentation of the name Rita Schmutlzer is Rita Schmutzler.

Publisher's note Springer Nature remains neutral with regard to jurisdictional claims in published maps and institutional affiliations.

The online version of the original article can be found at https://doi.org/ $10.1007 / \mathrm{s} 12687-018-0362-8$

Anne Brédart

anne.bredart@curie.net

1 Institut Curie, Supportive Care Department, Psycho-Oncology Unit, 26 rue d'Ulm, 75005 Paris, Cedex 05, France

2 University Paris Descartes, 71 avenue Edouard Vaillant, 92774 Boulogne-Billancourt, France

3 Université de Lorraine, 2LPN-CEMA, 23 boulevard Albert 1er-BP, 60446-54001 Cedex Nancy, France

4 Centre for Cancer Genetic Epidemiology, Department of Public Health and Primary Care, Worts Causeway, CB1 8RN, University of Cambridge, Cambridge, UK

5 Institut Curie, Cancer genetic clinic, 26 rue d'Ulm, 75005 Paris, Cedex 05, France

6 Department of Medical Genetics, University of Cambridge, Box 238, Level 6 Addenbrooke's Treatment Centre Cambridge Biomedical Campus, Cambridge CB2 0QQ, UK
7 Department of Clinical Genetics, Laboratory Medicine, Office for Medical Services and Department of Clinical Genetics, Lund University, 22185 Lund, Sweden

8 Netherlands Cancer Institute, Division of Molecular Pathology, Plesmanlaan 121, 1066, CX Amsterdam, The Netherlands

9 CESP, University Paris-Sud, UVSQ, INSERM, University Paris-Saclay, 16 avenue Paul Vaillant-Couturier, 94807 Villejuif, France

10 Familial Breast and Ovarian Cancer Centre, Cologne University, Hospital and Faculty of Medicine, Kerpener Str. 34, I 50931 Cologne, Germany

11 Department of Human Genetics, Department of Pathology, Leiden University Medical Centre, S4-P, P.O. Box 9600, 2300 RC Leiden, The Netherlands 